\title{
Quantum electron confinement in closely matched metals: Au films on $\operatorname{Ag}(111)$
}

\author{
D. Topwal, ${ }^{1,2, *}$ U. Manju, ${ }^{1,3,4}$ D. Pacilé,,${ }^{5,6}$ M. Papagno, ${ }^{6}$ D. Wortmann, ${ }^{7}$ G. Bihlmayer, ${ }^{7}$ S. Blügel, ${ }^{7}$ and C. Carbone ${ }^{6}$ \\ ${ }^{1}$ CSIR-Central Glass and Ceramic Research Institute, 196 S. C. Raja Mullik Road, Kolkata 700032, India \\ ${ }^{2}$ Sincrotrone Trieste, Strada Statale 14 Km 163.5, I-34149 Trieste, Italy \\ ${ }^{3}$ International Center for Theoretical Physics (ICTP), I-34014 Trieste, Italy \\ ${ }^{4}$ TASC Laboratory, IOM-CNR, Strada Statale 14 Km 163.5, I-34149 Trieste, Italy \\ ${ }^{5}$ Dipartimento di Fisica, Università della Calabria, 87036 Arcavacata di Rende (CS), Italy \\ ${ }^{6}$ Istituto di Struttura della Materia, Consiglio Nazionale delle Ricerche, Trieste, Italy \\ ${ }^{7}$ Peter Grünberg Institut and Institute for Advanced Simulation, Forschungszentrum Jülich and JARA, D-52425 Jülich, Germany
}

(Received 13 April 2012; published 9 August 2012)

\begin{abstract}
We examined by two-dimensional photoemission band mapping the electronic structure of Au films epitaxially grown on an $\mathrm{Ag}(111)$ substrate. The very similar structural and electronic properties of the two metals make this system extremely unfavorable for the occurrence and observation of electron confinement effects. At variance with previous spectroscopic studies, we show that the electron reflectivity at the interface sustains the formation of well-defined $s p$-derived quantum well states (QWS) and weak quantum well resonance states (QWRS) in the $\mathrm{Au}$ layers. The character and degree of confinement of these states are analyzed and quantitatively related to the $\mathrm{Au} / \mathrm{Ag}$ interface reflectivity on the basis of density functional theory (DFT) band structure calculations.
\end{abstract}

DOI: 10.1103/PhysRevB.86.085419

PACS number(s): 73.21.Fg, 79.60.Dp

\section{INTRODUCTION}

A quantum well confines into two or one dimensions particles which are originally free to move in space. The surface and interface potentials of an ultrathin film, supported by a substrate, can behave like the reflecting walls of a twodimensional (2D) quantum well for the electron waves. Electron confinement gives rise to a series of discrete QWS, with quantized momentum along the surface normal direction. ${ }^{1}$ Fully localized QWS form in the film if the electron energy corresponds to an energy gap of the substrate band structure. This is the typical case in semiconductor superlattices where the fundamental gaps of the semiconductors play the role of a barrier for the carrier confinement. ${ }^{2-4}$ An analogous situation is found in metallic systems, in correspondence with symmetry-related gaps extending over specific wave vector-energy regions. Fully confined QWS can form in a film within those regions when they cannot couple for symmetry reasons to other degenerate substrate states. ${ }^{5,6}$

More generally, a partial electron confinement in metallic films can be attained to various degrees depending on the matching conditions between film and substrate states. ${ }^{1,7}$ It has been shown that QWS or partially confined QWRS form rather commonly in metal films. ${ }^{8-11}$ Like in a classical Fabry-Perot interferometer, the amplitude of the confined electron waves can be described by their reflection and transmission occurring at the interface between the media. When the two materials have a very similar band structure, the potential change at the interface is minimal and the reflection is negligible. In such a case the interface is almost transparent for the propagation of the electron waves and common bands form between the film and the substrate.

Among the metals, $\mathrm{Au}$ and $\mathrm{Ag}$ have the same crystal structure, nearly perfectly matched lattices, and very similar band structures. Thus, the Au-Ag system is conceivably one of the worst candidates for the formation and observation of QWS and QWRS. Previous investigations proved that well defined QWS and QWRS exist in Ag films grown on $\mathrm{Au}(111)$ only in a very small energy range at the center of the Brillouin zone, in correspondence or in very close proximity to a gap of the 2Dprojected Au bulk bands. ${ }^{12,13}$ The Ag $s p$ bands, otherwise very similar to those of $\mathrm{Au}$, are slightly offset near the Fermi level to higher energy with respect to the Au states. In the reversed system, Au films on $\mathrm{Ag}(111)$, the Au $s p$ states are practically degenerate everywhere with substrate states and can couple with them for any energy-momentum state. Photoemission studies of Au films on $\mathrm{Ag}(111)$ substrate indeed did not observe any QWS or QWRS in the direction normal to (111) surface and concluded that the Au $s p$ states cannot be confined. ${ }^{12,14}$ This is therefore considered to be a paradigmatic case where reflectivity across the metal-metal interface is negligible.

In the present work we investigate electron confinement effects in Au films grown on $\mathrm{Ag}$ (111) substrate, performing a full $2 \mathrm{D}$ band mapping by means of angle resolved photoemission spectroscopy (ARPES). We report the observation of welldefined Au QWS and weak QWRS originating from bands with various characters. Detailed theoretical calculations taking into account the hybridization of Au $s p$ states with the semi-infinite $\operatorname{Ag}(111)$ substrate closely reproduce the experimental observations. The experimental and theoretical results show that the reflectivity across the Au-Ag interface is sufficient to localize QWR and QWRS over the whole energy and momentum region spanned by the Au $s p$ electrons. The character of the Au film states is found to vary from very faint resonances, due to a very weak but finite reflectivity, to fully confined states, depending on their symmetry and binding energy.

\section{EXPERIMENTAL AND COMPUTATIONAL DETAILS}

The experiments were carried out at the VUVphotoemission beamline of the Elettra Synchrotron Light Source, Italy, using a Scienta R-4000 electron analyzer. Valence band photoemission spectra were collected with a photon energy of $57 \mathrm{eV}$. Total energy and angular resolutions were $45 \mathrm{meV}$ and $0.015 \AA^{-1}$, respectively. Prior to the deposition of Au films, the $\mathrm{Ag}(111)$ substrate was prepared by 
cycles of $\mathrm{Ar}^{+}$ion sputtering and annealing to $750 \mathrm{~K}$. The clean $\operatorname{Ag}(111)$ surface showed a sharp $(1 \times 1)$ low energy electron diffraction (LEED) pattern with very low background and no presence of $\mathrm{C}$ and $\mathrm{O}$ signals in the core-level photoemission spectra. Thin Au films were then produced by in situ deposition onto the $\mathrm{Ag}(111)$ substrate held at $150 \mathrm{~K}$, followed by a slow warming up to $323 \mathrm{~K}$. Core-level spectra did not show any changes of spectral shapes and relative intensity of the $\mathrm{Au} 4 f$ and Ag $3 d$ levels before and after annealing, indicating that no intermixing occur between $\mathrm{Au}$ and Ag. ${ }^{12,15}$ All photoemission data reported here were obtained at $150 \mathrm{~K}$. Constant energy cut at the Fermi energy (i.e., Fermi surface) were acquired by collecting photoemission intensity maps over an azimuthal angular range of $60^{\circ}$ in steps of $1^{\circ}$ between the $\bar{\Gamma}-\bar{M}$ and the $\bar{\Gamma}-\bar{M}^{\prime}$ symmetry directions, and then symmetrizing them to get full two-dimensional contours.

DFT calculations were performed using the Jülich FLEUR code. ${ }^{16}$ On the basis of the full-potential linearized augmented plane wave method ${ }^{17}$ and the Greens function embedding method, ${ }^{18}$ this code allows not only the efficient treatment of transition metal surfaces and interfaces but also calculates transport properties at truly semi-infinite surfaces. The key quantity to enable the comparison to experimental data is the Bloch spectral function $A(\varepsilon, \vec{k})$, which can be understood as the energy- and momentum-resolved density of states. Neglecting any matrix element effects, this quantity is expected to resemble the ARPES data. To mimic the surface sensitivity of the photoemission process, we calculated the Bloch spectral function projected onto the surface atoms of our system with exponentially decaying contributions from deeper layers. To achieve a realistic simulation, proper treatment of the semiinfinite substrate is crucial, which ensures that a continuum of states is simulated. Within this continuum the truly localized states appear as $\delta$-function-like peaks, while resonances can be identified by broader structures, indicating a finite lifetime. Hence, localized states at the surface or in an adlayer can be identified easily. ${ }^{19}$ To compare with the experimental data, we calculated the Bloch spectral function of a setup consisting of 12 monolayers (ML) of $\mathrm{Au}$ attached to a semi-infinite $\mathrm{Ag}(111)$ substrate. The interface $\mathrm{Ag}$ was included in the surface calculation directly, while the effect of the remaining substrate was taken into account by the embedding self-energy, which is a property of bulk Ag. An averaged in-plane (fcc) lattice constant of $4.085 \AA$ has been assumed with an interlayer spacing of 2.355 and $2.359 \AA$ for $\mathrm{Au}$ and $\mathrm{Ag}$, respectively. For the exchange and correlation potential we used the local density approximation. ${ }^{20}$ The proper treatment of relativistic effects by including the spin-orbit coupling was necessary to obtain a good agreement for the bandwidth of the $d$ states and to obtain a Rashba-type spin-orbit splitting of the surface states. Apart from this, spin-orbit coupling does not significantly affect the transmission properties of the $\mathrm{Au} / \mathrm{Ag}(111)$ interface.

In the second setup we calculated the transmission properties for electrons across the $\mathrm{Au}-\mathrm{Ag}$ interface by considering semi-infinite Au attached to semi-infinite Ag. Using a transport code implementing the Landauer-Büttiker formalism ${ }^{21}$ we obtained the total transmission probability of electrons impinging on the interface for each energy and $k$ point $T\left(\varepsilon, \vec{k}_{||}\right)=\sum_{i}\left|t_{i}\right|^{2}$. In the case of a single band, the calculated transmission equals the square of the transmission amplitude, which is directly related to the reflectivity of the interface as $1=|t|^{2}+|r|^{2}$ and hence provides a measure for the localization of a quantum well resonance formed in the $\mathrm{Au}$ film. In particular, in a situation with perfect transmission the reflectivity will vanish and no resonance is formed, while zero transmission will lead to the formation of completely localized quantum well states.

\section{RESULTS AND DISCUSSION}

Figure 1(a) shows the band dispersions along the $\bar{K}-$ $\bar{\Gamma}-\bar{M}$ direction for a $12 \mathrm{ML}$ of $\mathrm{Au}$ film deposited on $\mathrm{Ag}(111)$ substrate, measured in form of intensity map by angle resolved photoemission spectroscopy. The most prominent spectral features in this figure are the parabolic band centered around $\bar{\Gamma}$ just below the Fermi energy $\left(E_{F}\right)$, corresponding to the $\mathrm{Au}(111)$ Shockley surface state, and bands mostly of $d$ character in the energy region beyond $2 \mathrm{eV}$ binding energy. The intensity map displays only faint features within $2 \mathrm{eV}$ from $E_{F}$. Energy distribution curves (EDCs) corresponding to the $\bar{\Gamma}-$ $\bar{M}$ direction are plotted in Fig. 1(b). Here the normal emission spectrum correspond to the one reported in literature ${ }^{12,13}$ and does not display any additional prominent features besides the Shockley surface state. However, a careful examination of the EDCs in the $\bar{\Gamma}-\bar{M}$ direction reveals very weak and broad structures dispersing towards $E_{F}$ for increasing emission angle. Moreover, relatively sharper and well-defined features, highlighted by green arrows, are observed near the high-symmetry point $\bar{M}$. The different spectral intensity of the states suggests that the confinement of the electrons in Au film on $\operatorname{Ag}(111)$ occurs to a widely different extent, depending on the electron energy and wave vector. It appears to be higher for the $s p$ bands near the $\bar{M}$ zone boundary, a fact that can be related to the presence of a gap around this high-symmetry point in the surface projected Ag band structure.

In order to enhance the very faint features present in the spectra, we normalize the area enclosed by the EDCs in
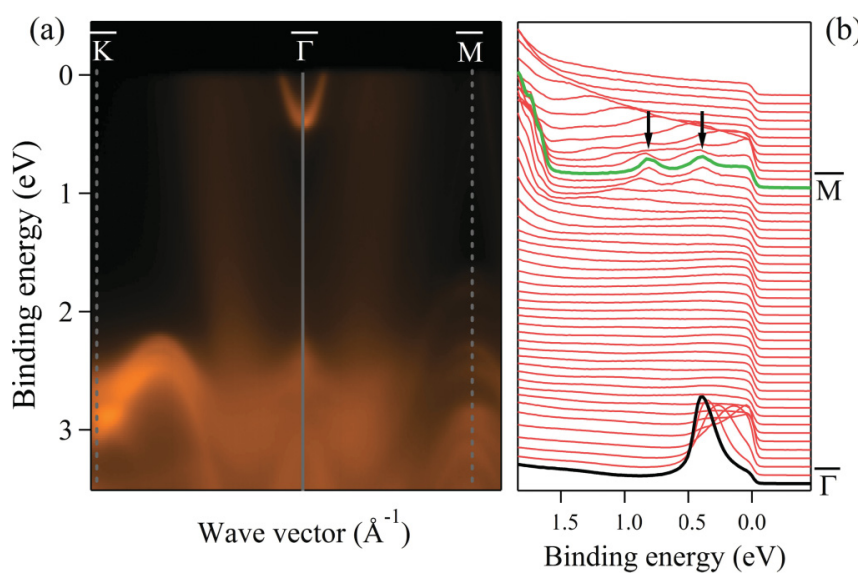

FIG. 1. (Color online) (a) Photoemission intensity map as obtained along the $\bar{K}-\bar{\Gamma}-\bar{M}$ direction for $12 \mathrm{ML}$ Au film deposited on $\mathrm{Ag}(111)$. (b) EDCs corresponding to the $\bar{\Gamma}-\bar{M}$ direction, plotted after normalizing the area enclosed by EDCs in the binding energy region between 1 and $2 \mathrm{eV}$. Arrows highlight two prominent, downward dispersing bands. 

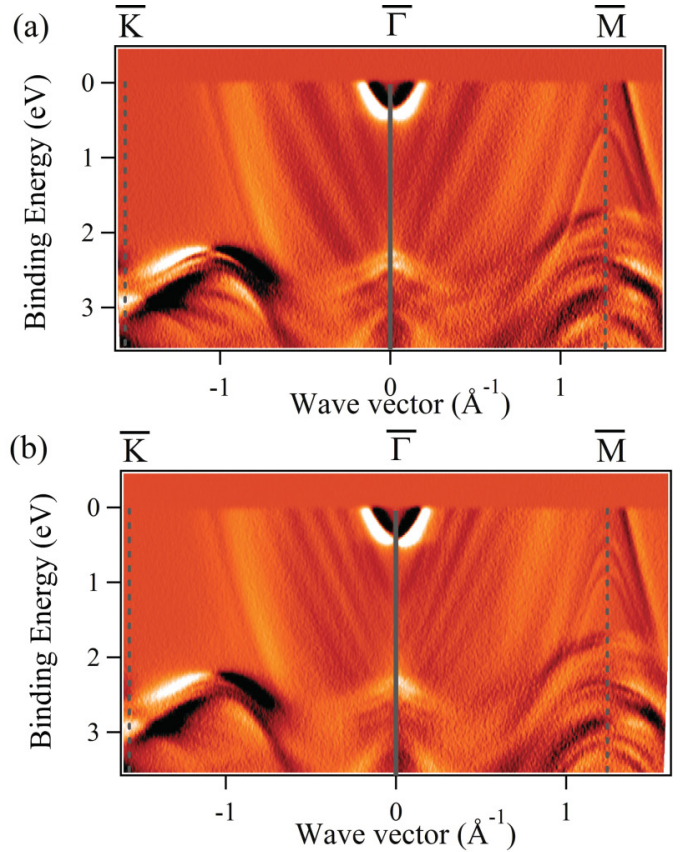

(c)

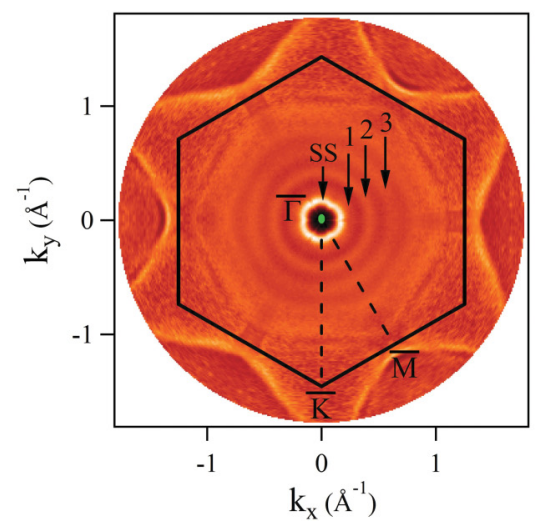

FIG. 2. (Color online) First derivative of the intensity map plotted along the $\bar{K}-\bar{\Gamma}-\bar{M}$ direction for (a) $7 \mathrm{ML}$ and (b) $12 \mathrm{ML}$ of $\mathrm{Au}$ film deposited on $\operatorname{Ag}(111)$. (c) Constant energy cuts at $E_{F}$ for $10 \mathrm{ML}$ $\mathrm{Au}$ film grown on $\mathrm{Ag}(111)$ substrate. Black hexagon marks the first Brillouin zone of Au, while series of QWRS contours and SS around $\bar{\Gamma}$ are also marked.

the binding energy region between 1 and $2 \mathrm{eV}$ and plot in Figs. 2(a) and 2(b) the first derivative of the intensity maps measured for $\mathrm{Au}$ film of 7 and $12 \mathrm{ML}$ thicknesses along the $\bar{K}-\bar{\Gamma}-\bar{M}$ direction. Weaker features that could not be otherwise observed now become clearly visible. The data display a series of strongly dispersive Au bands that can be attributed to QWRS, with minima at $\bar{\Gamma}$. Electrons, only marginally confined in the Au film, form over a wide energy range weak QWRS, despite the energy overlap between Ag and Au bands. As indicated by the DFT calculation discussed in the following, a very small but finite electrons' reflectivity at the $\mathrm{Au} / \mathrm{Ag}$ interface support the formation of such QWRS, although the crystal structure, lattice parameters, and band structure of two metals are very similar.

Unlike other QWRS in metallic films on metal and semiconducting substrates, ${ }^{22,23}$ these QWRS display a nearly freestanding character, possibly due to the very close resemblance of $\mathrm{Ag}$ and $\mathrm{Au}$ bands. The number of QWRS and their relative positions in energy and momentum space depends, as expected, on the Au film thickness, as it can be seen comparing the 7 and $12 \mathrm{ML}$ data. For binding energies beyond $2 \mathrm{eV}, \mathrm{Au}$ film states of mostly $d$-like character ${ }^{24}$ are observed. These states are closely spaced in energy due to the weak dispersion of the $d$ bands. The higher spectral intensity of the $d$-derived states, compared to the $s p$ bands, can be considered a mark of stronger confinement. This can be understood from the different energy positions of the $d$ bands in $\mathrm{Ag}$ and $\mathrm{Au}$ : While in $\mathrm{Ag}$ the $d$ states are located well above $4 \mathrm{eV}$ binding energy, due to scalar-relativistic effects in Au these states are found at smaller binding energies. Hence for a thin $\mathrm{Au}$ film on $\mathrm{Ag}, \mathrm{Au} d$ states cannot couple to the Ag substrate bands and remain localized.

Further information on the character of the quantum confined states is provided by the Fermi energy contours, shown in Fig. 2(c) for a $10 \mathrm{ML}$ Au film. The innermost and the most prominent circular state near the $\bar{\Gamma}$ point arise from the Au surface state. A series of concentric circular quantum well states around the $\bar{\Gamma}$ point closely correspond to the expected contours of the Au $s p$-derived states. ${ }^{22}$ The $n=1,2$, and 3 bands as well as the SS are nearly isotropic about the center of the surface Brillouin zone. States with higher $n$ exhibit a hexagonal-like energy contour with corners lying along the $\bar{\Gamma}-\bar{M}$ axis of $\mathrm{Au}(111)$ surface Brillouin zone, because of the gap opening occurring at the $\bar{K}$ point.

The experimental findings can be analyzed with the help of the DFT calculations. Figures 3(a) and 3(b) show the experimental results over an extended energy range and the corresponding Bloch spectral function of $12 \mathrm{ML}$ of $\mathrm{Au}$ film on a semi-infinite $\operatorname{Ag}(111)$ substrate along the $\bar{K}-\bar{\Gamma}-\bar{M}$ direction for comparison. The unique feature of our band structure calculation is the treatment of the semi-infinite substrate, which ensures that a continuum of states is simulated and therefore localized states at the surface or in the film can be identified. Correspondingly, the value of the Bloch spectral function is visualized by the color code/intensity scale, which enables a simple comparison with the experimental observation. ${ }^{19}$ The calculations closely reproduce the band dispersion of $s p$ and $d$ states presented in Figs. 2(b) and 3(a). The surface states and the Au $d$ bands appear as the most intense features in the theory as well as in the experiment. The electronic structure of the $d$ states in the $2-6 \mathrm{eV}$ energy range is dominated by relatively sharp bands, due to substantial energy difference between the $\mathrm{Ag} 4 d$ and the Au $5 d$ states leading to strong confinement and formation of QWS in Au. Two other notable features are the formation of quantum well states in the lower part of the $s p$ band beyond approximately $7 \mathrm{eV}$ binding energy, at the $\bar{\Gamma}$ point and, especially, some weak features in the $s p$ band within $2 \mathrm{eV}$ from the Fermi level. Note that in the lower part of the $s p$ band the bottom six bands are rather sharp, while the bands in between 7 and $8 \mathrm{eV}$ are broadened due to the overlap with the $\operatorname{Ag} s$ band. The sharp parabolic band at $7 \mathrm{eV}$ is a surface state that is also clearly seen in the ARPES [Fig. 3(a)] and was found in a DFT calculation earlier. ${ }^{25}$

To investigate the origin of the faint features closer to the Fermi level in more detail, we compare in Figs. 4(a) and 4(b) the Bloch spectral function and the corresponding transmission map calculated for an interface between semi-infinite $\mathrm{Au}$ and semi-infinite Ag in the (111) direction. Although the Au and Ag band structures are similar in band character and the 


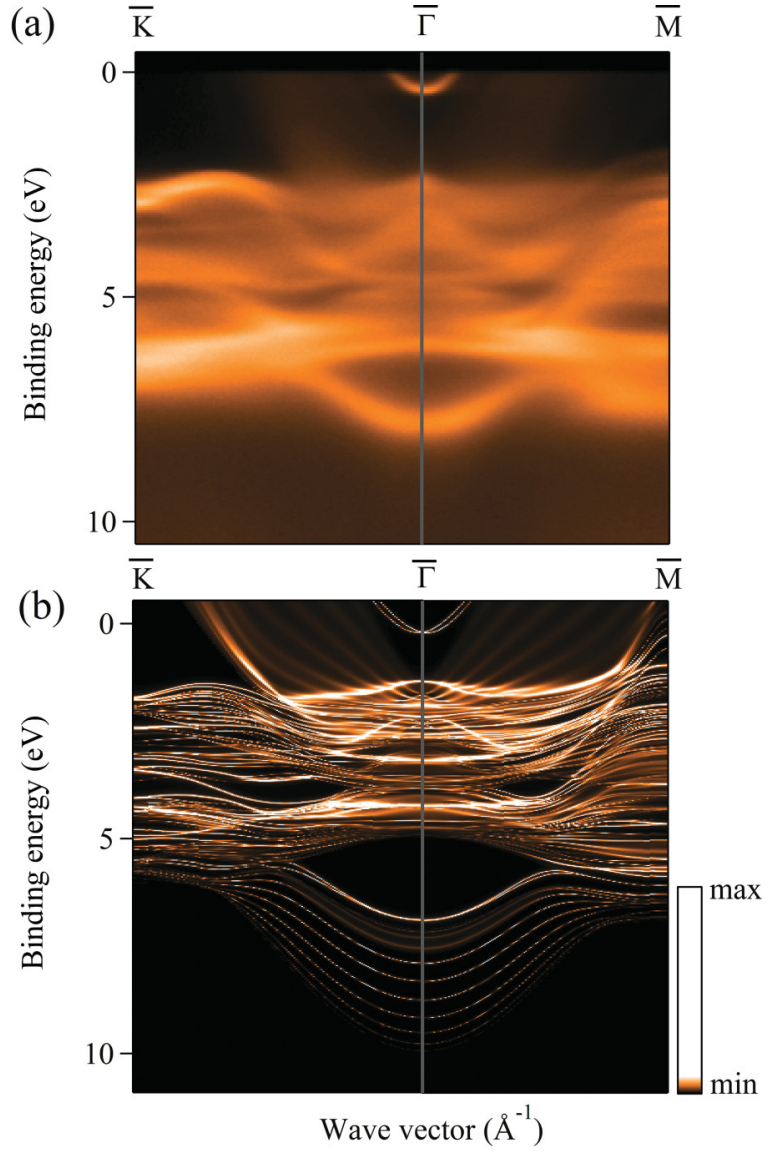

FIG. 3. (Color online) (a) Photoemission intensity maps for 12 ML $\mathrm{Au}$ film grown on $\mathrm{Ag}(111)$ substrate. (b) Calculated Bloch spectral function for a 12 ML Au film on semi-infinite $\operatorname{Ag}(111)$.

bands in this energy region are strongly degenerate, the Au/Ag interface does not show perfect transmission $T^{2}$ (which would lead to an integer transmission, corresponding to the number of bands projected to this particular area in 2D $k$ space). There exists a small but finite electron reflectivity $\left(R^{2}=1-T^{2}\right.$, between 0 and 1), which leads to the formation of weak QWRS in the Au film, though the transmission for most of the investigated $k$ and energy is very close to unity. In the energy and $k$ range in which $s p$-like states are present in both $\mathrm{Au}$ and $\mathrm{Ag}$, the reflectivity is extremely small, being close to 0.02 . It is this very small, but finite value of the reflectivity which leads to the formation of the experimentally observed weak QWRS. The calculated QWRS bands are very weakly localized in the $\mathrm{Au}$ film, as indicated by the color code in Fig. 4. The typical linewidth of these resonance states is around $0.3 \mathrm{eV}$. The lifetime corresponding to the localization of the electron is about $1 \mathrm{fs}$. On the other hand, the group velocity of $\mathrm{Au}$ electrons close to the Fermi level (at a $k$ point $10 \%$ of the line along the $\bar{\Gamma}-\bar{M}$ direction) is of the order $2 \mathrm{~nm} / \mathrm{fs}$. Hence the time required for the electron to travel through the $\mathrm{Au}$ film is significantly longer (approximately $8 \mathrm{fs}$ ) demonstrating the weak confinement. The expected exceptions are located close to $\bar{\Gamma}$ and at higher energies in the energy-momentum space around $\bar{M}$ and near $\bar{K}$ where no Bloch states are present in the semi-infinite substrate. This explains the formation of prominent $\mathrm{Au}$ surface state and sharp and intense features

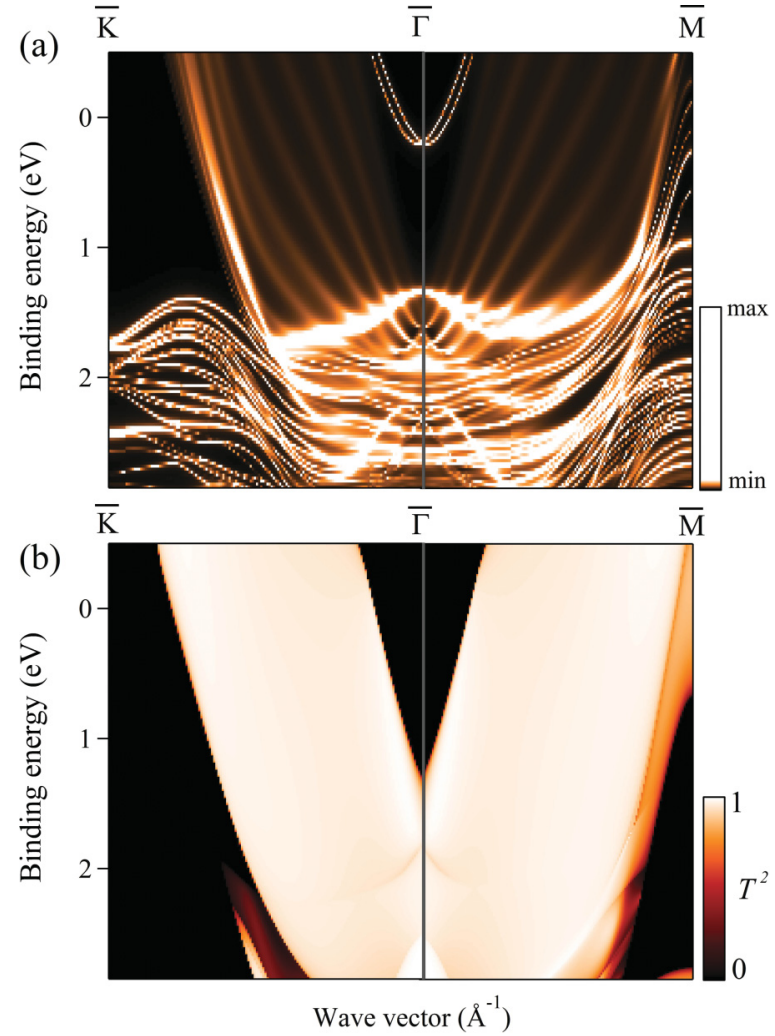

FIG. 4. (Color online) (a) Bloch spectral function for a 12 ML Au film on semi-infinite $\operatorname{Ag}(111)$ close to the Fermi-level. (b) Transmission at a $\mathrm{Ag} / \mathrm{Au}(111)$ interface in the same energy range.

in these gaps. As observed in the experimental spectra, the resonances are rather flat in this energy range. At an energy of about $1 \mathrm{eV}$ a change in slope can be observed in the dispersion of these resonances, which is in agreement with the earlier measurements on Au QWS. ${ }^{25}$ At energies higher that $2 \mathrm{eV}$ we observe a very structured transmission map resulting from symmetry selection rules of the states of different characters that meet in this region.

\section{CONCLUSION}

In conclusion, we report the observation of quantum confined states in Au films grown on an $\mathrm{Ag}(111)$ substrate. The results emphasize that electron quantum confinement is a quite general property of metal film and multilayer systems, even for closely matched elements. Despite the very similar structural and electronic properties of the two metals, angle resolved photoemission shows that electron confinement gives raise to well-defined QWS and to weak QWRS in the full energy range spanned by the Au $s p$-derived states. The experimental results are understood with the help of DFT calculations which relate the degree of confinement to the finite electronic reflectivity across the $\mathrm{Au} / \mathrm{Ag}(111)$ interface. The theoretical data show that a reflectivity of a few percent at the interface is sufficient to ensure the formation of observable QWRS.

\section{ACKNOWLEDGMENT}

U. Manju thanks ICTP, Trieste, Italy for the TRIL fellowship. 
*dinesh.topwal@trieste.ism.cnr.it, dinesh.topwal@gmail.com

${ }^{1}$ T.-C. Chiang, Surf. Sci. Rep. 39, 181 (2000).

${ }^{2}$ I. Matsuda, T. Ohta, and H. W. Yeom, Phys. Rev. B 65, 085327 (2002).

${ }^{3}$ S.-J. Tang, L. Basile, T. Miller, and T.-C. Chiang, Phys. Rev. Lett. 93, 216804 (2004).

${ }^{4}$ S.-J. Tang, W.-K. Chang, Y.-M. Chiu, H.-Y. Chen, C.-M. Cheng, K.-D. Tsuei, T. Miller, and T.-C. Chiang, Phys. Rev. B 78, 245407 (2008).

${ }^{5}$ M. A. Mueller, T. Miller, and T.-C. Chiang, Phys. Rev. B 41, 5214 (1990).

${ }^{6}$ T. Valla, P. Pervan, M. Milun, A. B. Hayden, and D. P. Woodruff, Phys. Rev. B 54, 11786 (1996).

${ }^{7}$ W. E. McMahon, T. Miller, and T.-C. Chiang, Phys. Rev. B 54, 10800 (1996).

${ }^{8}$ D. V. Vyalikh, Yu. Kucherenko, F. Schiller, M. Holder, A. Kade, S. L. Molodtsov, and C. Laubschat, Phys. Rev. B 76, 153406 (2007).

${ }^{9}$ A. M. Shikin, D. V. Vyalikh, Yu. S. Dedkov, G. V. Prudnikova, V. K. Adamchuk, E. Weschke, and G. Kaindl, Phys. Rev. B 62, R2303 (2000).

${ }^{10}$ A. M. Shikin, O. Rader, G. V. Prudnikova, V. K. Adamchuk, and W. Gudat, Phys. Rev. B 65, 075403 (2002).

${ }^{11}$ J. J. Paggel, T. Miller, and T.-C. Chiang, Science 283, 1709 (1999).

${ }^{12}$ T. Miller, A. Samsavar, G. E. Franklin, and T.-C. Chiang, Phys. Rev. Lett. 61, 1404 (1988).
${ }^{13}$ Li Huang, X. G. Gong, E. Gergert, F. Forster, A. Bendounan, F. Reinert, and Zhenyu Zhang, Europhys. Lett. 78, 57003 (2007).

${ }^{14}$ F. J. Palomares, M. Serrano, A. Ruiz, F. Soria, K. Horn, and M. Alonso, Surf. Sci. 513, 283 (2002).

${ }^{15}$ R. J. Culbertson, L. C. Feldman, P. J. Silverman, and H. Boehm, Phys. Rev. Lett. 47, 657 (1981); T. C. Hsieh, T. Miller, and T.-C. Chiang, Phys. Rev. B 33, 2865 (1986); T. C. Hsieh and T.-C. Chiang, Surf. Sci. 166, 554 (1986).

${ }^{16}$ For a program description see http://www.flapw.de.

${ }^{17}$ E. Wimmer, H. Krakauer, M. Weinert, and A. J. Freeman, Phys. Rev. B 24, 864 (1981).

${ }^{18}$ D. Wortmann, H. Ishida, and S. Blügel, Phys. Rev. B 66, 075113 (2002).

${ }^{19}$ P. Moras, D. Wortmann, G. Bihlmayer, L. Ferrari, G. Alejandro, P. H. Zhou, D. Topwal, P. M. Sheverdyaeva, S. Blügel, and C. Carbone, Phys. Rev. B 82, 155427 (2010).

${ }^{20}$ S. H. Vosko, L. Wilk, and M. Nusair, Can. J. Phys. 58, 1200 (1980).

${ }^{21}$ Y. Imry, and R. Landauer, Rev. Mod. Phys. 71, S306 (1999)

${ }^{22}$ P. Moras, D. Topwal, P. M. Sheverdyaeva, L. Ferrari, J. Fujii, G. Bihlmayer, S. Blügel, and C. Carbone, Phys. Rev. B 80, 205418 (2009).

${ }^{23}$ N. J. Speer, S.-J. Tang, T. Miller, and T.-C. Chiang, Science 314, 804 (2006).

${ }^{24}$ D.-A. Luh, J. J. Paggel, T. Miller, and T.-C. Chiang, Phys. Rev. Lett. 84, 3410 (2000).

${ }^{25}$ R. Mazarello, A. Dal Corso, and E. Tosatti, Surf. Sci. 602, 893 (2008). 